\title{
Nähe auf Distanz - sensible Gestaltung von Kommunikationstechnik
}

\author{
Thies Schneider ${ }^{1}$, Marc Hassenzahl ${ }^{1}$, Eva Lenz ${ }^{1}$, Kirstin Kohler ${ }^{2}$, Wasili \\ Adamow $^{2}$, Patrick Beedgen ${ }^{2}$ \\ Fachbereich Gestaltung I Erlebnis und Interaktion, Folkwang Universität der Künste ${ }^{1}$ \\ Fakultät für Informatik I User Experience \& Interaction Design, Hochschule Mannheim²
}

\section{Zusammenfassung}

Wir beschreiben an einem Beispiel, wie mit Hilfe von Technik Verbundenheitspraktiken unterstützt, erweitert oder neue Praktiken etabliert werden können. Dafür werden fünf Familien, jeweils bestehend aus drei Generationen, umfassend betrachtet und mögliche Veränderungspotentiale identifiziert. Diese werden dann in Konzepte übersetzt und als funktionale Prototypen mindestens zehn Tage im Alltag der Familien erprobt. Am Beispiel von Familie Maier und ihrem Fenster zeigen wir, wie Konzepte basierend auf dem detaillierten Verständnis der Familienstruktur erarbeitet werden und welche Wirkung diese Konzepte auf die entsprechenden Familien haben können.

\section{Nähe, Verbundenheit und Technik}

Sich anderen Menschen nahe zu fühlen, also das Bedürfnis nach „Verbundenheit" und „Nähe” zu befriedigen, ist zentral für Wohlbefinden und Lebenszufriedenheit (z.B. Ryan \& Deci 2000). Eine dafür wichtige soziale Struktur ist die Familie. Familien entwickeln eigene Praktiken, die Nähe schaffen, wie beispielsweise das gemeinsame Abendessen. Ist physische Anwesenheit nicht gegeben, kann familiäre Nähe über die Distanz mit Hilfe von Technik erreicht werden. Videotelefonie ist hier stark verbreitet (Kirk et al. 2010; Judge et al. 2010). Diese Arbeiten verfolgen meist den Ansatz, Familien dabei zu beobachten oder zu befragen, wie sie Videotelefonie nutzen. Daraus werden generalisierte Schlüsse zur Verbesserung der Technik gezogen. Dabei bleiben die individuellen Konstellationen und Praktiken der Familien unberücksichtigt. Eine Ausnahme stellen Miller und Sinanan (2014) dar, die vom Einzelfall ausgehend zeigen, wie unterschiedlich die Praktiken sind, die aus der gleichen Technik entstehen. In dieser Arbeit spielt die Gestaltung der Technik allerdings keine Rolle.

Das BMBF-geförderte Projekt „Nähe auf Distanz" stellt einzelne Familien und deren Bedürfnisse nach Nähe in den Mittelpunkt. In dem laufenden Projekt werden neue familiäre Praktiken der Nähe erarbeitet und mit Hilfe gängiger Technik in funktionale Prototypen überführt. Ziel 
ist es, in einem iterativen und nutzerzentrierten Prozess, bedeutungsvolle Interaktionen zwischen den Familienmitgliedern in den Vordergrund zu stellen und nicht die zugrundeliegende Technik. Wir folgen dabei Miller und Sinanan (2014), die betonen, dass technikvermittelte Kommunikation weder vermittelter noch "unnatürlicher" ist als Kommunikation von Angesicht zu Angesicht. Sie ist nur anders und dieses Anderssein ist eine Folge der genauen Ausgestaltung der Situation und damit auch der verwendeten Technik. Ein Beispiel ist die Tatsache, dass Videotelefonie, wie Skype, dem Sprecher oft das eigene Bild ebenso zeigt, wie das des Gegenübers. Dies ist Angesicht zu Angesicht kaum möglich. Die Tatsache, dass man sich selbst beim Sprechen aus der Perspektive des Gegenübers sehen kann, ist zunächst lediglich eine technische Funktion. Sie führt allerdings zu einer verstärkten Ich-Wahrnehmung und beeinflusst damit auch Verhalten, Kommunikation und Erleben. Gestalterisch stellt sich nun nicht die Frage, ob diese Funktion prinzipiell "gut", "schlecht", "natürlich" oder "unnatürlich" ist, sondern, ob sie in einer bestimmten Situation eingesetzt werden kann, um gewünschte Erlebnisse zu unterstützen. Eine solche Gestaltung ist ganzheitlich und psychologisch informiert (Hassenzahl 2010; Neustaedter et al. 2013).

Im Folgenden wird das methodische Vorgehen kurz erläutert und anschließend an einer der teilnehmenden Familien beispielhaft dargestellt. Ziel ist es zu zeigen, wie umfassend selbst bereits gängige Technik in den Lebensalltag einzugreifen vermag und wie wichtig es ist, die Wirkung von Technik und ihre Individualisierbarkeit schon bei der Gestaltung mitzudenken (oder wie hier zum Gestaltungsgegenstand zu machen) und permanent im Auge zu behalten.

Für das Projekt haben sich fünf Familien mit insgesamt 23 Personen zur Verfügung gestellt. Jede Familie ist auf mindestens zwei Haushalte verteilt und hat jeweils drei Generationen. Die gestaltungsorientierte Handlungsforschung (Sein et al. 2011) gibt uns die Möglichkeit, mit der Unterschiedlichkeit innerhalb der Familien und den individuellen Veränderungen, die durch die einzelnen Konzepte initiiert werden, umzugehen. Der enge Kontakt, den wir zu den teilnehmenden Familien haben, ist Voraussetzung für Offenheit und die lebensweltliche Relevanz der Konzepte. Um auf die Familien eingehen zu können und die Familienstruktur zu verstehen, werden verschiedene empirische Methoden eingesetzt, Konzepte permanent reflektiert und an die Belange der Familien und der Forscher/Gestalter angepasst. Durch Tagebücher und leitfadengestützte Einzelinterviews erhalten wir detaillierte Kenntnisse über die Beziehungen innerhalb der Familie. Diese wurden in Gestaltungsworkshops eingebracht, um so familienspezifische Konzepte zu entwickeln, die dann in Prototypen umgesetzt wurden. Ein Testzeitraum von mindestens zehn Tagen ermöglicht das Erfassen der Auswirkungen auf den Lebensalltag und die sozialen Prozesse innerhalb der Familie.

\section{Das Fenster als Beispiel für vermittelte Nähe}

Das im Folgenden präsentierte Beispiel basiert auf den realen Bedürfnissen und Praktiken der Familie Maier (Namen verändert). Familie Maier lebt mit vier Personen in zwei Haushalten wenige Kilometer voneinander entfernt. Großmutter Marie (62) wohnt alleine in einer großen Wohnung im Stadtzentrum. Louise (32), Maries Tochter, wohnt mit ihrem Mann Peter (34) und dem gemeinsamen zweijährigen Sohn Max in einer kleinen Wohnung in einem Vorort. 
Marie hat eine besonders enge Bindung zu ihrer Tochter und möchte auch für ihren Enkel eine wichtige Rolle spielen. Gleichzeitig will sie möglichst eigenständig bleiben. Louise arbeitet einmal pro Woche als Krankenschwester. Ihre freie Zeit verbringt sie mit Max oder sie geht mit ihm zu ihrer Mutter. Ihr Mann Peter arbeitet fünf Tage pro Woche im Schichtdienst. Dadurch ist er nur wenig in den Alltag eingebunden. Für das Konzept relevant ist die sehr unterschiedlich empfundene Nähe innerhalb der Familie. Während Marie und Louise eine überdurchschnittlich harmonische und enge Beziehung pflegen, werden die anderen Familienmitglieder oft von der Kommunikation ausgeschlossen. Dies zeigt sich insbesondere bei den ausführlichen und häufigen Telefonaten. Da Max noch nicht telefonieren kann, ist er aufgrund fehlender Aufmerksamkeit dann laut und quengelig. Peter hingegen zieht sich aus den Gesprächen zurück und verliert dadurch den Anschluss an das Familiengeschehen. Peter steht dem eher ratlos gegenüber, während Marie distanziert bleibt.

Das Fenster nimmt die enge Beziehung zwischen Marie und Louise als Ausgangspunkt und soll das "hermetische" Kommunikationsverhalten für die anderen Familienmitglieder öffnen. Wie eingangs erwähnt, ist die Gestaltung der Interaktionen der Schlüssel zur Veränderung der Nähepraktiken (Konzeptvideo: https://vimeo.com/132535275). Wie ein architektonisches Fenster ist es örtlich gebunden. Durch Schieberegler lassen sich die Fenster öffnen und eine Verbindung initiieren.

Nur wenn beide Fenster geöffnet sind, entsteht eine Verbindung. Das stellt die Autonomie des jeweiligen Haushalts sicher. Um bei geschlossenem Fenster trotzdem einen Kontakt zu initiieren ist es möglich, am Rahmen des Fensters anzuklopfen. Das Klopfmuster wird auf der anderen Seite mechanisch reproduziert. Da das Fenster als dedizierte Verbindung zwischen Haushalten und nicht als intime Kommunikation zwischen Personen angelegt ist, sollten Max und Peter mehr Gelegenheit finden, sich an Maries und Louises Kommunikation zu beteiligen. Als weitere Modifikation wurde in dem Rahmen eine Weitwinkellinse über die Kamera geschoben, um mehr vom Raum zusehen, die Intimität eines Gesprächs aufzubrechen und es für andere zu öffnen.

Das Fenster wurde in beiden Haushalten installiert und den Teilnehmern für zehn Tage überlassen. Innerhalb dieser Zeit wurde das Fenster 66 Mal aktiviert. Durchschnittlich wurde es pro Aktivierung zwei Minuten genutzt (Gesamtaktivierungsdauer 144 Minuten). In Tagebüchern und darauf folgenden Interviews erfassten wir die geänderten Praktiken und die empfundene Nähe der Teilnehmer. Es hat eine Veränderung der Kommunikation stattgefunden. Alltägliche Dinge, die schnell abgeklärt werden konnten, wurden überwiegend über das Fenster besprochen. Eine neue Form des Austauschs mit der ganzen Familie fand zwischendurch „zum Spaß“ statt. Durch das Fenster hat Marie Max öfter gesehen als sonst und glaubte, präsenter für ihren Enkel zu sein. Sie fühlte sich durch die Kommunikation über das Fenster Max näher. Ihren Schwiegersohn sah sie auch häufiger. Durch die Gestaltung der Interaktion war es Max möglich, ohne seine Mutter die Großmutter über das Fenster zu kontaktieren. So konnten wir mit dem Konzept die Kommunikation für andere Familienmitglieder ein wenig öffnen. Allerdings muss man auch festhalten, dass das Fenster von Mutter und Tochter immer noch zu sehr als direkter Kommunikationskanal verstanden wurde. Zwar wurden intime Dinge nicht über das Fenster besprochen (dafür wurde dann das Telefon genutzt), die kurze mittlere Aktivierungszeit weist aber darauf hin, dass das Fenster nicht einfach aufgemacht wurde und die 
Haushalte ihren Alltag teilten. Es wurde immer explizit kommuniziert und wie vorher meist von Mutter und Tochter initiiert. Während das Fenster zu ein wenig mehr "Öffentlichkeit" in der Familie der Tochter führte, war es dem Telefon noch zu ähnlich, um neue, inklusivere Kommunikationspraktiken zu stimulieren.

Die erste Iteration zeigt deutlich, wie grundlegend die Möglichkeiten von sorgfältig konfigurierter Technik sind, Alltagskommunikation zu verändern und wie wichtig kleinste Gestaltungsentscheidungen, wie z.B. ein Schieber für die Kamera, sein können. Das vorliegende Beispiel und der zugrundeliegende explorative Prozess zeigen, wie sensible Technikgestaltung aus unserer Sicht sein sollte: Ausgehend vom Einzelfall und mit Gespür für den Effekt, den Technik in ihren verschiedenen Konfigurationen auf alltägliche Praktiken und Erlebnisse haben kann. Während eine technikorientierte Sicht oft immer noch die technische Innovation in den Vordergrund stellt und wenig Gespür für die potentiell großen Wirkungen vermeintlich kleiner Gestaltungsunterschiede zeigt, halten wir ein Umdenken für nötig. Uns scheint es an technischen Möglichkeiten nicht zu mangeln, dafür aber an Wissen, wie diese Technik im Alltag innovativ und wohlbefindenorientiert eingesetzt werden kann.

\section{Danksagung}

Finanziert durch das BMBF-Projekt „Nähe auf Distanz“ (FKZ: 16SV7093K)

\section{Literaturverzeichnis}

Hassenzahl, M. (2010). Experience Design: Technology for All the Right Reasons. San Rafael, CA: Morgan \& Claypool.

Judge, T. K., Neustaedter, C., \& Kurtz, A. F. (2010). The family window: the design and evaluation of a domestic media space. Proceedings of the SIGCHI Conference on Human Factors in Computing Systems (CHI '10). ACM, New York, NY, USA, S. 2361-2370.

Kirk, D. S., Sellen, A., \& Cao, X. (2010). Home video communication: mediating 'closeness'. Proceedings of the 2010 ACM conference on Computer supported cooperative work (CSCW '10). ACM, New York, USA, S. 135-144.

Miller, D. \& Sinanan, J. (2014). Webcam. Cambridge: Polity Press.

Neustaedter, C., Harrison, S., \& Sellen, A. (2013). Connecting Families - Introduction. In C. Neustaedter, S. Harrison \& A. Sellen (Eds.), Connecting Families: The Impact of New Communication Technologies on Domestic Life. London: Springer. (S. 1-14).

Ryan, R. M. \& Deci, E. L. (2000). Self-determination theory and the facilitation of intrinsic motivation, social development, and well-being. American Psychologist, 55(1), S. 68-78.

Sein, M., Henfridsson, O., Purao, S., Rossi, M., \& Lindgren, R. (2011). Action design research. MIS Quarterly, 35(1), S. 37-56. 